\title{
Unusual Isolated Complete Tear of Subscapularis and Biceps Long Head Tendon Associated with Heterotopic Ossification
}

\author{
Hoon-Sang Sohn, Duck In Baek, Min Soo Shon \\ Department of Orthopedic Surgery, Center for Joint Surgery, National Medical Center, Seoul, Korea
}

\begin{abstract}
Heterotopic ossification $(\mathrm{HO})$ within the substance of the subscapularis tendon is a rare lesion which remains a poorly described condition with little known of the exact mechanisms involved. Furthermore, its clinical importance remains still unclear. To our knowledge, there are no studies present to data regarding $\mathrm{HO}$ within the substance of the subscapularis tendon, even with resultant isolated complete tear of the subscapularis tendon. Here we present a case of huge $\mathrm{HO}$ associated with unusual isolated complete tear of subscapularis tendon concomitant with tear of biceps long head tendon. After arthroscopic debridement for the complete tear site of biceps long head tendon, mini-open excision of the ossification and subscapularis repair with suture anchor fixation were performed. The patient showed complete recovery of strength and function of the subscapularis at subsequent 24 months follow up.
\end{abstract}

(Clin Shoulder Elb 2014;17(3):127-133)

Key Words: Heterotopic ossification; Coracoid impingement; Isolated subscapularis tear

Heterotopic ossification $(\mathrm{HO})$ of the shoulder has been found in associated with traumatic brain injury, spinal cord injury, local trauma such as fracture or dislocation, and shoulder surgery. ${ }^{1,2}$ However, $\mathrm{HO}$ in the rotator cuff is an extremely rare lesion which remains a poorly described condition with little known of the exact mechanisms involved. Furthermore, although some authors have reported that large ossification or calcification within the substance of the subscapularis tendon is a rare cause of subcoracoid stenosis and coracoid impingement, ${ }^{3-5)}$ its clinical importance remains still unclear. To our knowledge, there are no studies present to data regarding $\mathrm{HO}$ within the substance of the subscapularis tendon, even with resultant isolated tear of the subscapularis tendon. Here we present a case of huge $\mathrm{HO}$ associated with unusual isolated complete tear of subscapularis tendon and biceps long head tendon, and successfully treated with mini-open excision of the ossification and subscapularis repair with suture anchor fixation. The authors were conducted after obtaining approval from the Institutional Review Board at our institution for this case report.

\section{Case Report}

A 45-year-old male, who was a sedentary (office) worker, was referred to our outpatient department with pain and tenderness on the anterior aspect of the right, dominant shoulder. He used to play tennis every weekend for more than 10 years, but 1 month ago he injured his right shoulder during a powerful forward smashing with a popping sound. Although the patient suffered from right deep and anterior shoulder pain and painful limitation of the shoulder motion, he continued to play tennis lightly and anterior shoulder and mid-arm pain developed once again 3 days before visiting to our clinic. He denied history of previously other trauma or any shoulder pain.

Inspection of right shoulder revealed slight dimpling and ecchymosis is shown in the proximal portion of the biceps, known as Popeye deformity around the right arm. Physical evaluation showed restriction of active and passive motion: external rotation was $30^{\circ}$ and internal rotation till the level of L4-5. Clinical testing of the subscapularis tendon revealed a positive Lift-off, Belly press, and Bear hug test with muscle strength 4 of 5 , and

Received November 29, 2013. Revised April 19, 2014. Accepted April 22, 2014.

Correspondence to: Min Soo Shon

Department of Orthopedic Surgery, National Medical Center, 245 Eulji-ro, Jung-gu, Seoul 100-799, Korea

Tel: +82-2-2262-4707, Fax: +82-2-2278-9570, E-mail: smsice125@naver.com

Financial support: None. Conflict of interests: None. 

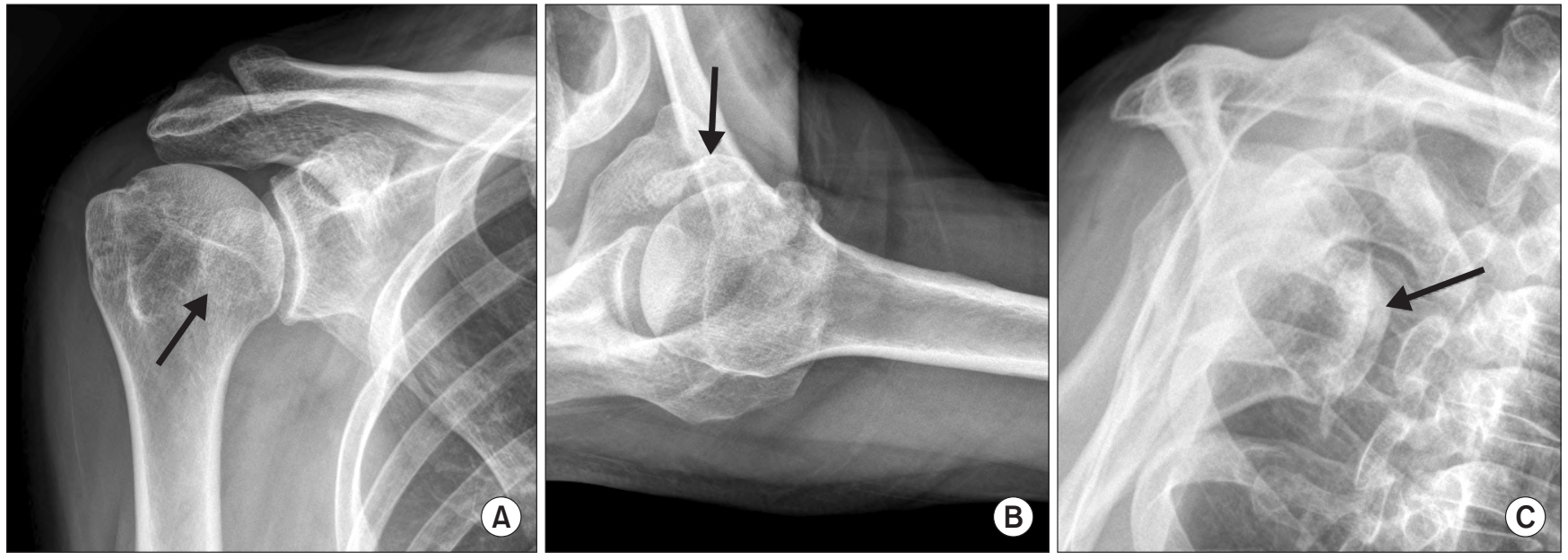

Fig. 1. A huge radio-opaque lesion (arrows) was shown under the coracoid process on the lesser tuberostiy on preoperative. True anteroposterior (A), axillary (B), and outlet views $(\mathrm{C})$.

moreover, coracoid impingement and cross-arm adduction test were provoked as well. Plain radiographs showed a huge, ovoid radio-opaque lesion on the anterior aspect with a diameter of about $2.7 \times 3.0 \mathrm{~cm}$, which was suspected to be the subscapularis tendon (Fig. 1).

A magnetic resonance arthrography was performed to clarify the lesion more accurately, and a huge mass-like lesion was seen within the substance of the subscapularis tendon. The magnetic resonance (MR) findings was not shown the findings of the acute avulsion fracture of the lesser tuberosity, represented as linear bone marrow edema (with decreased signal intensity on T1weighted and increased signal intensity on T2-weighted images) as well as a decreased to absent signal along the fracture line. Signal intensity of the lesion was very similar to bone on T1- and T2-weighted images, which was consistent with findings of ossification, unlike calcification (Fig. 2). The ossified lesion occupied almost all of subscapularis tendon and measured about $26 \times 30$ $\mathrm{mm}$ of longest diameter and $4.8 \mathrm{~mm}$ of thickness on axial and sagittal images. The shortest distance between the ossification within the subscapularis tendon and the tip of coracoid process was about $3.1 \mathrm{~mm}$ on the axial image.

The subscapularis tendon was ruptured completely with fullthickness on the entire footprint, but was not retracted inferiorly or medially (Fig. 3). In addition, there was not shown findings, such as atrophy and fatty degeneration, represented chronicity of the subscapularis tear or old avulsion fracture of lesser tuberosity on preoperative MR images (Fig. 4).

The long head of biceps tendon was not observed on the axial image (Fig. 3). There was no supraspinatus or infraspinatus tendon tear on the poreoperative MR imaging.

The patient underwent general anesthesia and was placed in a lateral decubitus position with traction applied to the forearm. The standard posterior portal was produced, and the arthroscope was introduced into the glenohumeral joint. The arthroscopy confirmed a complete full-thickness tear of the subscapularis tendon laden with a huge and dense ossification, enveloped by the thin articular-side fibers within the substance of the subscapularis tendon and complete rupture of the biceps long head tendon (Fig. 5).

After arthroscopic examination and debridement for the complete tear site of biceps long head tendon, surgery was performed with a limited deltopectoral approach, because it is difficult to completely remove a huge ossification and repair a complete, full-thickness ruptured subscapularis tendon by using an arthroscope. A large ossified mass covered with a thin subscapularis tendon, which was not retracted medially, was identified. After turning it over to approach through more thinner articular side and to reserve relatively thick and healthy bursal side of the tendon, the ossified mass was removed from the substance of the subscapularis tendon completely (Fig. 6).

The insertion site on the lesser tuberosity is prepared to optimize the environment for tendon healing, the ruptured tendon was repaired back to the original footprint in 2 by 2 double-row configuration with bioabsorbable suture anchors.

An abduction brace was applied for 6 weeks after surgery. He was instructed to proceed with self-rehabilitation, which started with the passive forward flexion of the shoulder joint 4 weeks after surgery. Passive internal rotation was allowed 6 weeks, and the patient expressed pain relief 8 weeks postoperatively; visual analogue scale score 20 of 100 . At 12 weeks, we confirmed integrity of the repaired subscapularis tendon and no residual or recurred ossification (Fig. 7). The involved shoulder had recovered strength and the function of the subscapularis, including active internal rotation and passive external rotation, was restored. At his 24-month follow-up, the patient, who had not been seen since his prior 12 week clinic visit, had no pain, full range of mo- 

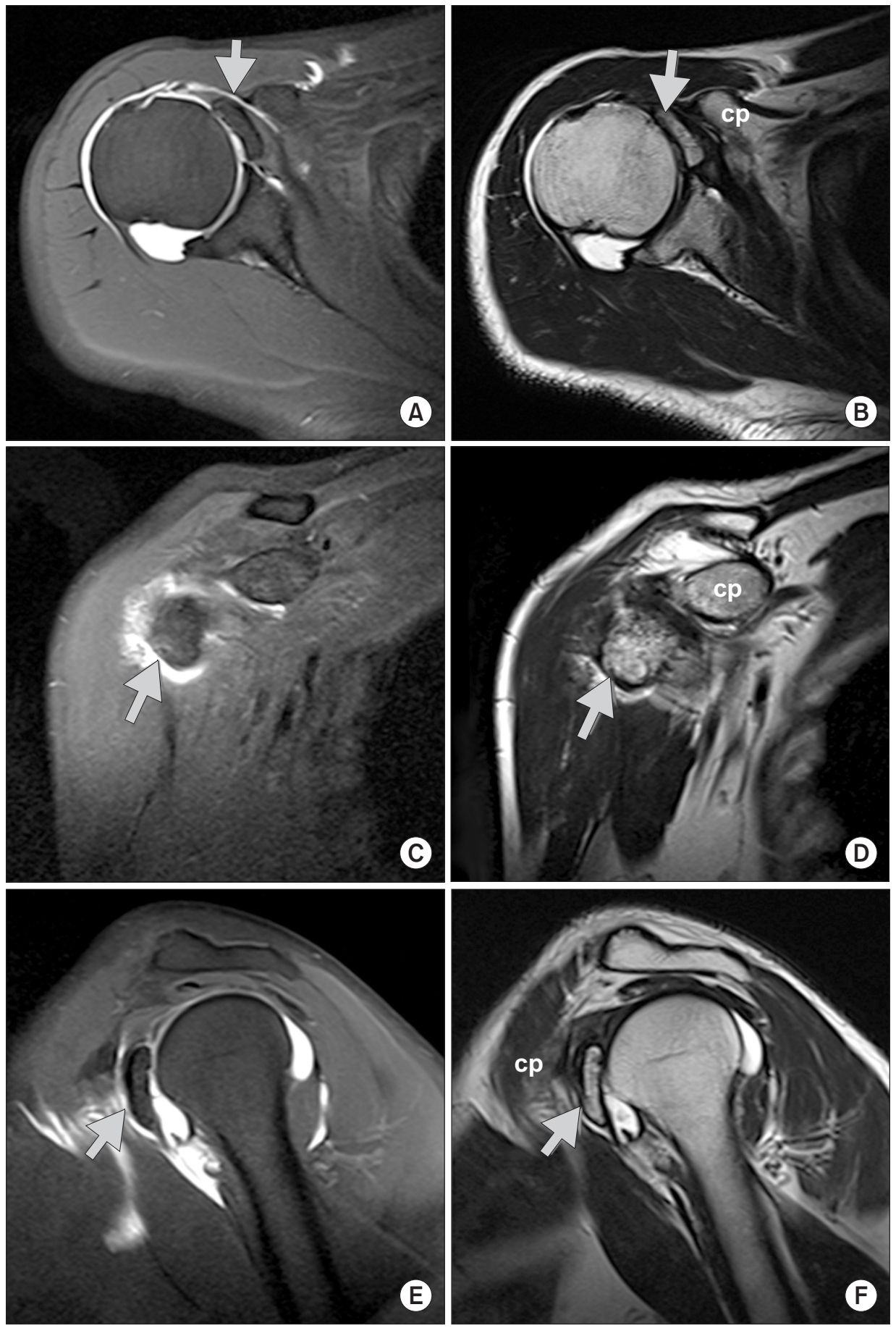

Fig. 2. Magnetic resonance arthrography of the right shoulder. The ossified mass (arrows) within the substance of the subscapularis tendon was shown and the subcoracoid space was narrowed. cp: coracoid process.

tion, and full strength.

\section{Discussion}

The subscapularis is the largest and most powerful of the rotator cuff muscles. In the shoulder, the subscapularis has an important role in shoulder motion, especially internal rotation, both active and passive mechanism for glenohumeral stability and coordinated cuff function as the "force couple". Several literatures have reported that $\mathrm{HO}$ in the rotator cuff has been caused by traumatic brain injury, severe burn, trauma, or unknown origin. ${ }^{1,45)}$ However, the etiology of tendon ossification in the subscapularis tendon is still poorly described and its clinical relevance also remains unclear. In our review of the literature, we found only two studies reporting $\mathrm{HO}$ within the subscapularis tendon. Busilacchi et al. ${ }^{4}$ reported the en bloc removal of 

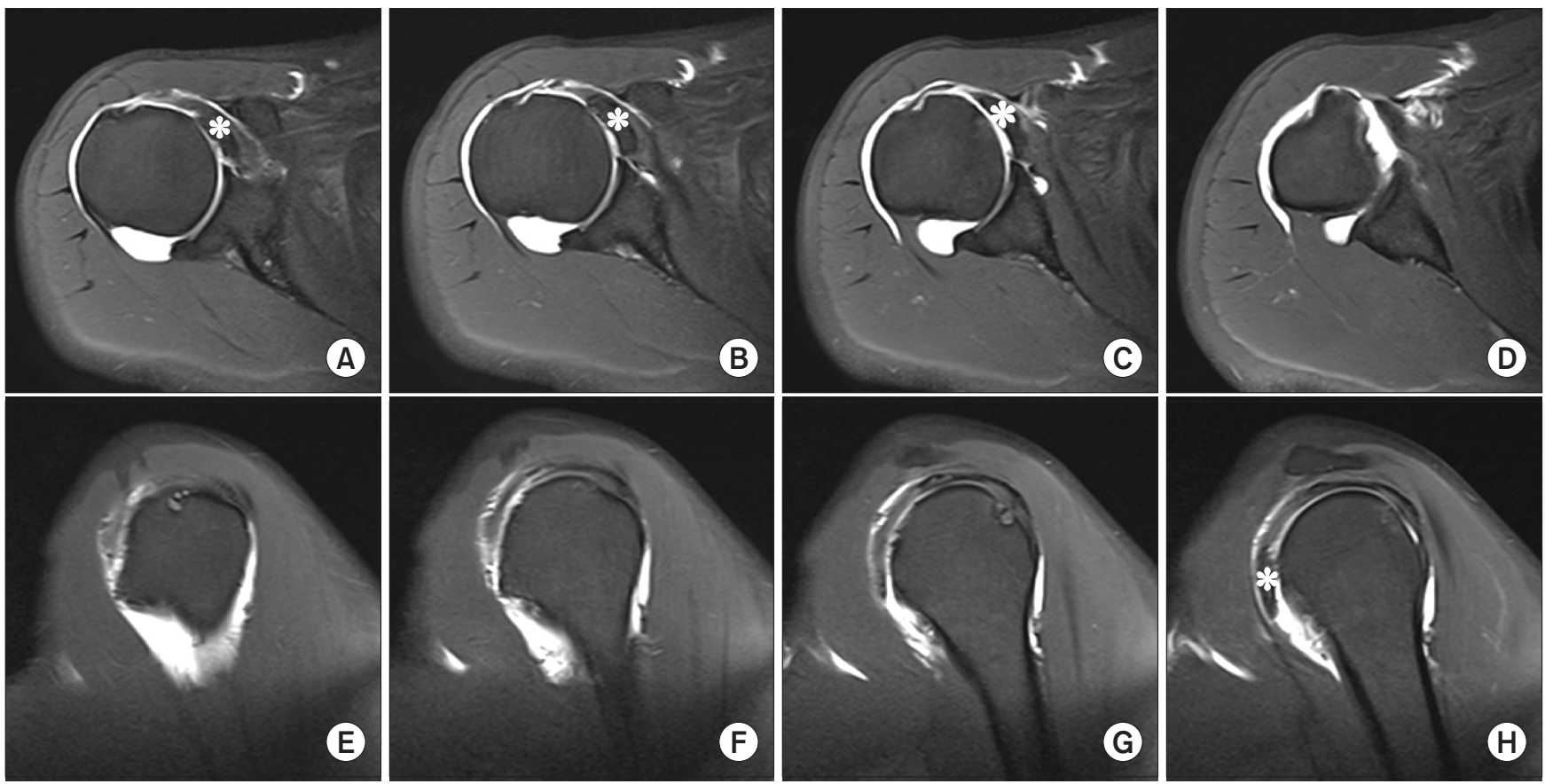

Fig. 3. (A) Tracing in the superior to inferior direction on axial T1-weighted and (B) lateral to medial direction on sagittal T1-weighted sequences with fat suppression, the subscapularis tendon was seen complete, full-thickness tear at just lateral of ossified mass (asterisks) without medial retraction, and the long head of biceps tendon was not observed on the axial image.
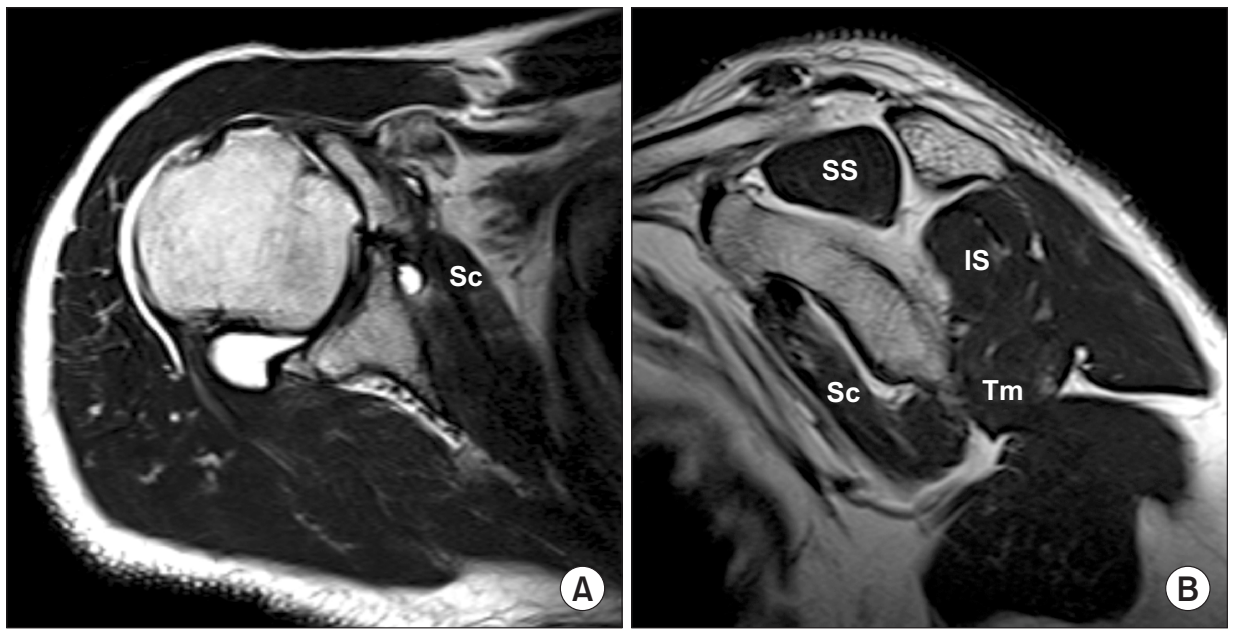

Fig. 4. Axial (A) and sagittal T1-weighted images (B) were not shown findings, such as atrophy and fatty degeneration, represented chronicity of the subscapularis tear. SS: supraspinatus, IS: infraspinatus, Sc: subscapularis, Tm: teres minor. a subscapularis tendon ossification which occurred in a patient with tuberculosis. Peidro et al. ${ }^{5)}$ described multiple ossifications of the subscapularis tendon accompanied by simultaneous subcoracoid impingement. However, no history of trauma was relevant to ossification and a subscapularis tendon tear was not noted in the two cases. To our best knowledge, the present case was first focused on isolated complete tear of subscapularis tendon associated with huge ossification within the substance of the subscapularis tendon.

Another injury considered in the differential diagnosis of shoulder pain in the present case is an isolated avulsion fracture of the lesser tuberosity. The isolated lesser tuberosity fracture is a very rare and frequently involve younger patients. The high percentage of the chronic cases suggests that they are often overlooked at the time of injury. Although old or neglected condition of avulsion lesser tuberosity fracture was little reported, previous reports revealed that clinical symptoms specific to chronic cases were chronic insidious and gradually aggravated pain, exacerbated with abduction and external rotation movements of the shoulder. ${ }^{6,7)}$ On radiographs, the fracture fragment in most cases of isolated avulsion fracture of lesser tuberosity is retracted more inferiorly and medially in the chronic setting or even in the acute 

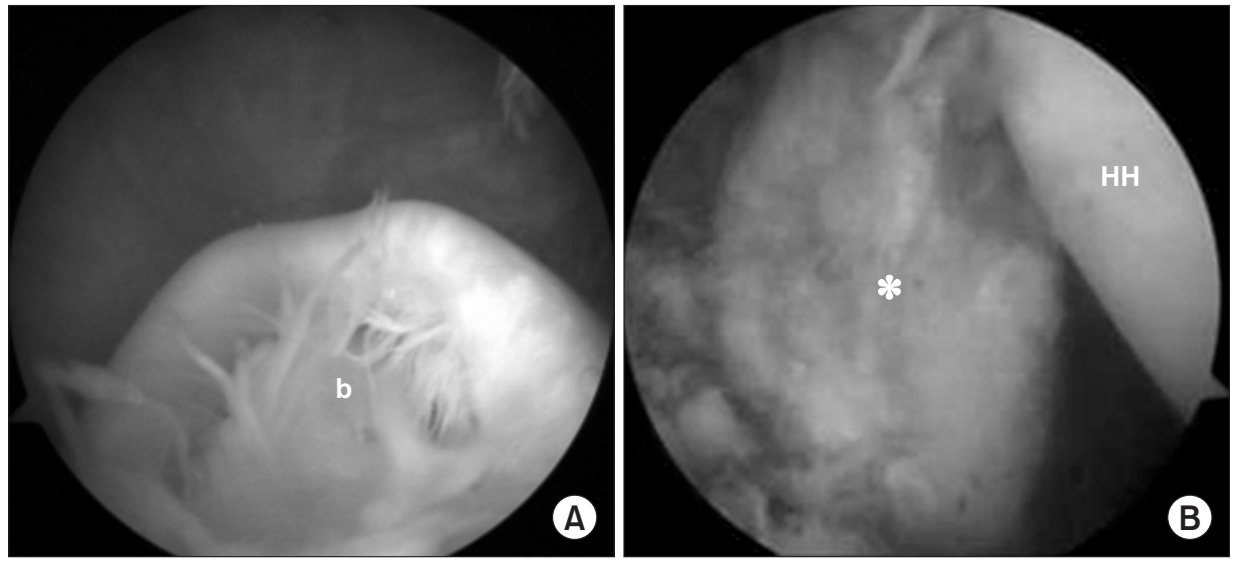

Fig. 5. Arthroscopic images of the right shoulder showing the frayed insertion site of complete ruptured biceps long head tendon at the superior glenoid labrum (A) and a fullthickness tear of the subscapularis tendon laden with a huge ossification (asterisk) from the posterior viewing portal with the patient in the lateral decubitus position (B). $\mathrm{HH}$ : humeral head, b: insertion site of completely ruptured biceps long head tendon.
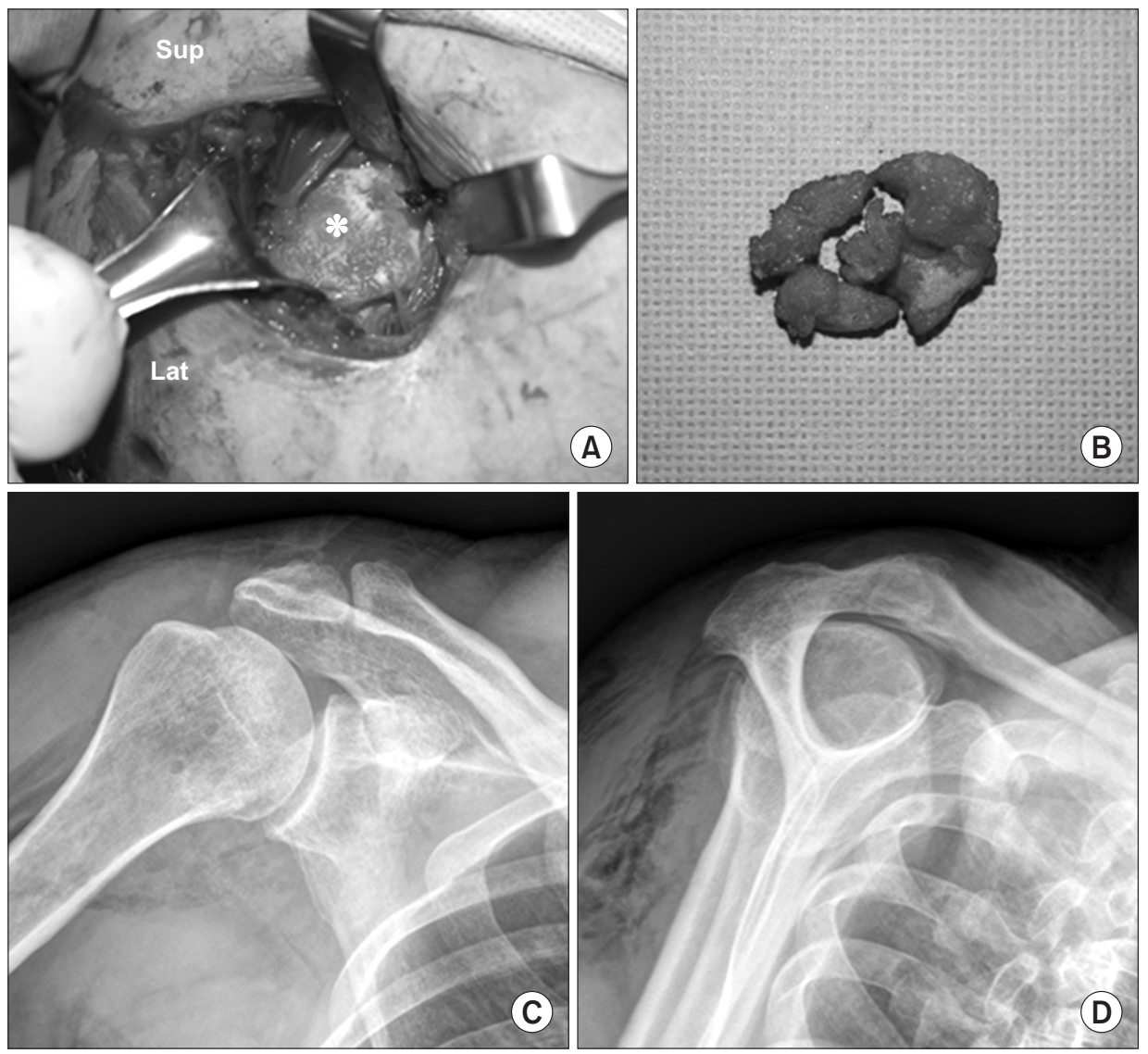

Fig. 6. (A) Intraoperative finding showing a large ossified mass (asterisk) covered with a thin subscapularis tendon. (B) The surgical specimens were removed from within the substance of the subscapularis tendon. Postoperative radiographs $(\mathrm{C}, \mathrm{D})$ of the right shoulder. A radio-opaque lesion disappeared completely. Sup: superior, Lat: lateral.

setting with large avulsion fragment. ${ }^{6,7)}$ Some authors reported the presence of the exostosis on the proximal humerus (as the result of enchondral ossification of the fracture hematoma) or fibrous band connected with ossified mass at the level of lesser tuberosity in patients with isolated avulsion fracture of lesser tuberosity in adolescent or skeletal immature periods." In addition, because the subscapularis tendon is directly attached to the fractured fragment, cases of chronic or neglected fracture of lesser tuberosity leads to an insufficiency of the musculotendinous unit of the subscapularis, which can be shown minimum grade
2 fatty infiltration of the subscapularis muscle and atrophy. ${ }^{6,7}$ Although the possibility of the old isolated fracture of lesser tuberosity could not be completely ruled out, there was no clinical feature and radiographic findings specific to chronic injury in this case.

Subscapularis tendon tear is usually chronic and degenerative in nature as a part of an anterosuperior or massive rotator cuff tear pattern and rarely isolated. Some authors have reported that large ossification or calcification within the substance of the subscapularis tendon is a rare cause of subcoracoid stenosis and 

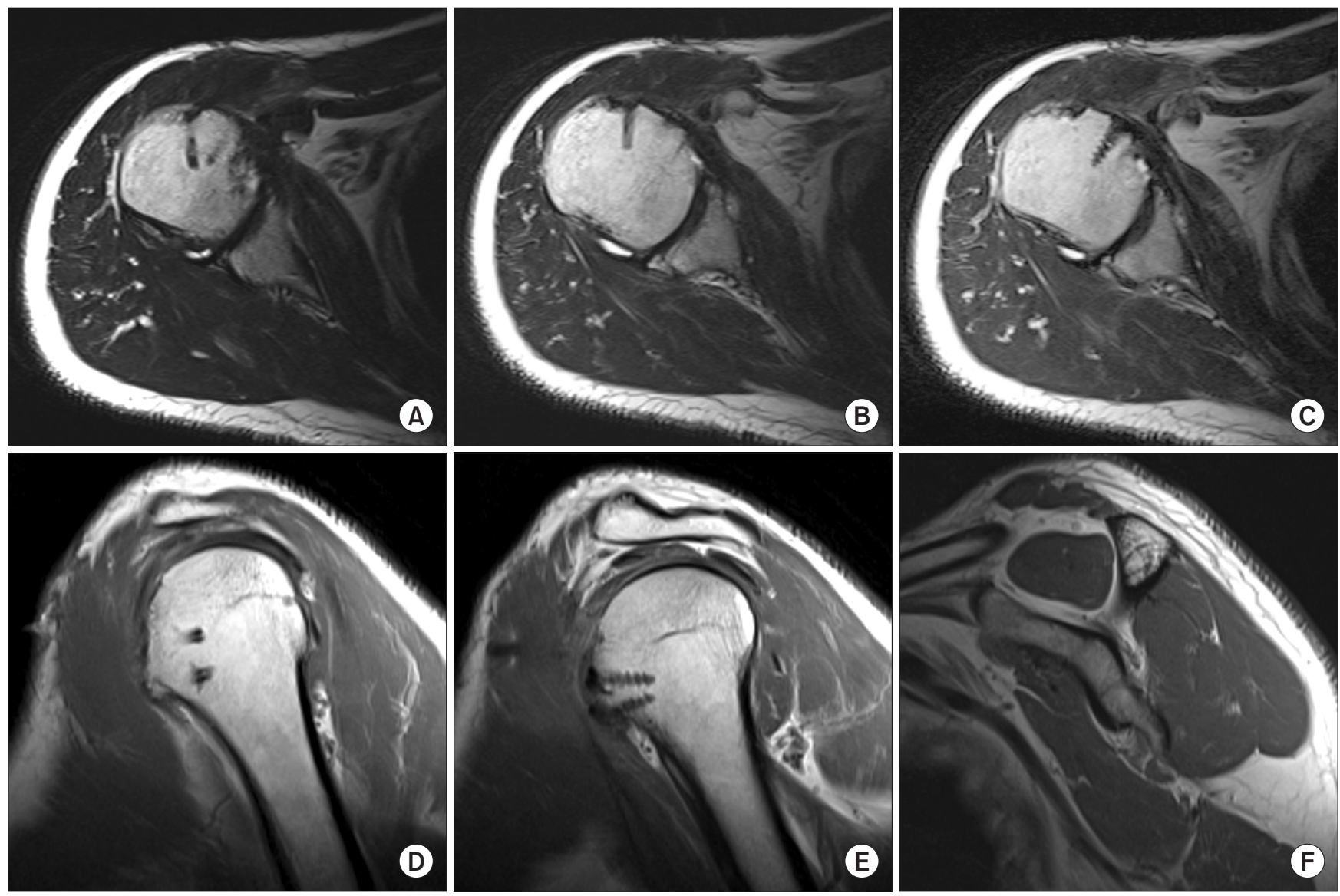

Fig. 7. At 12 weeks after surgery, postoperative axial (A-C) and sagittal (D-F) T2-weighted magnetic resonance imaging scans showed the repaired subscapularis tendon was maintained on the original footprint with a relative normal thickness.

coracoid impingement caused by an increase in its contents of that space. ${ }^{3-5)}$ These conditions have also been reported one of predisposing factors for the subscapularis tear. Gerber et al. ${ }^{8)}$ described the coracoid impingement as a primary contributor to anterior shoulder pain, as well as resultant pathology of the subscapularis tendon and the long head of the biceps tendon. Richards et al..$^{9)}$ reported a narrowed coracohumeral distance may be one of the possible causes of the subscapularis pathology. In this case, a 3.1-mm of the distance between the ossification and the coracoid process, as secondary subcoracoid impingement, might be related to the rupture of the subscapularis tendon as an extrinsic factor of the subscapularis pathology. Furthermore, the last traumatic event might aggravate or convert the preexisting rupture of subscapularis tendon into the complete full-thickness tear, although he denied previous shoulder pain. There are other possible factors affecting subscapularis tear in this case; abnormal shortening of tendon excusion or pathological weakening due to fibrosis and degeneration of the remnant tendon, or anterior instability. We believe that this ossification within the substance of the subscapularis tendon, for any reason, resulted in (or resulted from) isolated complete tear of the subscapularis tendon.
Because the subscapularis tendon is susceptible to marked medial migration, tendon retraction is another consequence of delayed presentation that enhances the difficulty of repair. ${ }^{10)}$ One of important points of this report highlights, even though a complete full-thickness tear of the subscapularis tendon was noted on the MR image, that retraction of the tendon did not occur and the entire length of the occupied tendon by ossified mass was maintained. We suggested that a huge ossified mass might have been caught up in the coracoid process or the glenoid rim, which in turn could have prevented the medial retraction of the subscapularis tendon.

Concomitant involvement of the biceps long head tendon with isolated subscapularis tendon tears occurs in 31\% to 56\% of cases and involves thickening, subluxation or dislocation, and rupture. ${ }^{11)}$ In this case, it is unclear whether the subscapularis tear or the biceps tendon tear was primary in causing these patient's symptoms. However, we also believe that $\mathrm{HO}$ within the subscapularis or isolated complete tear of subscapularis tendon negatively influenced biceps long head tendon, resulting in complete tear.

The present case is the first in the literature to describe a huge 
ossification within the substance of the subscapularis tendon, even with resultant isolated complete tear of the subscapularis tendon concomitant with biceps long head tendon tear. Although its cause was not clarified, tendon ossification in the subscapularis should be considered as a rare etiology of the isolated subscapularis tear and can be successfully treated with complete excision of the ossification and subscapularis repair.

\section{References}

1. Fuller DA, Mani US, Keenan MA. Heterotopic ossification of the shoulder in patients with traumatic brain injury. J Shoulder Elbow Surg. 2013;22(1):52-6.

2. Pape HC, Marsh S, Morley JR, Krettek C, Giannoudis PV. Current concepts in the development of heterotopic ossification. J Bone Joint Surg Br. 2004;86(6):783-7.

3. Arrigoni P, Brady PC, Burkhart SS. Calcific tendonitis of the subscapularis tendon causing subcoracoid stenosis and coracoid impingement. Arthroscopy. 2006;22(10):1139.e1-3.

4. Busilacchi A, Bottegoni C, Gigante A. Arthroscopic management of heterotopic ossification of the subscapularis tendon in a patient with tuberculosis: a case report. J Shoulder Elbow Surg. 2012;21(1):e1-5.
5. Peidro L, Serra A, Suso S. Subcoracoid impingement after ossification of the subscapularis tendon. J Shoulder Elbow Surg. 1999;8(2):170-1.

6. Klasson SC, Vander Schilden JL, Park JP. Late effect of isolated avulsion fractures of the lesser tubercle of the humerus in children. Report of two cases. J Bone Joint Surg Am. 1993;75(11):1691-4.

7. Goeminne S, Debeer P. The natural evolution of neglected lesser tuberosity fractures in skeletally immature patients. J Shoulder Elbow Surg. 2012;21(8):e6-e11.

8. Gerber C, Terrier F, Zehnder R, Ganz R. The subcoracoid space. An anatomic study. Clin Orthop Relat Res. 1987;(215): 132-8.

9. Richards DP, Burkhart SS, Campbell SE. Relation between narrowed coracohumeral distance and subscapularis tears. Arthroscopy. 2005;21(10):1223-8.

10. Lafosse L, Jost B, Reiland Y, Audebert S, Toussaint B, Gobezie R. Structural integrity and clinical outcomes after arthroscopic repair of isolated subscapularis tears. J Bone Joint Surg Am. 2007;89(6):1184-93.

11. Gerber $C$, Hersche $O$, Farron A. Isolated rupture of the subscapularis tendon. J Bone Joint Surg Am. 1996;78(7):1015-23. 